\title{
Anorectal Function After lleo-Rectal Anastomosis Is Better than Pelvic Pouch in Selected Ulcerative Colitis Patients
}

\author{
Maie Abdalla ${ }^{1,2}$ (D) $\cdot$ Rickard Norblad $^{3} \cdot$ Malin Olsson $^{3} \cdot$ Kalle Landerholm $^{4} \cdot$ Peter Andersson $^{5,6}$. \\ Johan D. Söderholm ${ }^{3} \cdot$ Roland Andersson ${ }^{4} \cdot$ Pär Myrelid ${ }^{3}$
}

Received: 25 February 2019 / Accepted: 20 July 2019 / Published online: 1 August 2019

(c) The Author(s) 2019

\begin{abstract}
Background With a lifelong perspective, $12 \%$ of ulcerative colitis patients will need a colectomy. Further reconstruction via ileo-rectal anastomosis or pouch can be affected by patients' perspective of their quality of life after surgery.

Aim To assess the function and quality of life after restorative procedures with either ileo-rectal anastomosis or ileal pouchanal anastomosis in relation to the inflammatory activity on endoscopy and in biopsies.

Method A total of 143 UC patients operated with subtotal colectomy and ileo-rectal anastomosis or pouches between 1992 and 2006 at Linköping University Hospital were invited to participate. Those who completed the validated questionnaires (Öresland score, SF-36, Short Health Scale) were offered an endoscopic evaluation including multiple biopsies. Associations between anorectal function and quality of life with type of restorative procedure and severity of endoscopic and histopathologic grading of inflammation were evaluated.

Results Some 77 (53.9\%) eligible patients completed questionnaires, of these 68 (88.3\%) underwent endoscopic evaluation after a median follow-up of 12.5 (range 3.5-19.4) years after restorative procedure. Patients with ileo-rectal anastomosis reported better overall Öresland score: median $=3$ (IQR 2-5) for ileo-rectal anastomosis $(n=38)$ and 10 (IQR 5-15) for pouch patients $(n=39)(p<0.001)$. Anorectal function (Öresland score) and endoscopic findings (Baron-Ginsberg score) were positively correlated in pouch patients (tau: $0.28, p=0.006$ ).

Conclusion Patients operated with ileo-rectal anastomosis reported better continence compared to pouches. Minor differences were noted regarding the quality of life. Ileo-rectal anastomosis is a valid option for properly selected ulcerative colitis patients if strict postoperative endoscopic surveillance is carried out.
\end{abstract}

Keywords Ulcerative colitis · Ileo-rectal anastomosis · Ileal pouch-anal anastomosis · Quality of life

$\begin{array}{ll}\text { Abbreviations } \\ \text { BG } & \text { Baron-Ginsberg score } \\ \text { CCGQoL } & \text { Cleveland clinic global quality of life score } \\ \text { IBD } & \text { Inflammatory bowel disease } \\ \text { IPAA } & \text { Ileal pouch-anal anastomosis } \\ \text { IRA } & \text { Ileo-rectal anastomosis } \\ \text { IQR } & \text { Interquartile range } \\ \text { OR } & \text { Odds ratio } \\ \text { SF-36 } & \text { Short form } 36 \text { questions } \\ \text { SHS } & \text { Short health scale }\end{array}$

Electronic supplementary material The online version of this article (https://doi.org/10.1007/s10620-019-05757-6) contains supplementary material, which is available to authorized users.

Maie Abdalla

maie.abdalla@liu.se

Extended author information available on the last page of the article

$\begin{array}{ll}\text { tau } & \text { Kendall-tau correlation coefficient } \\ \text { UC } & \text { Ulcerative colitis } \\ \text { VAS } & \text { Visual analogue scale } \\ \text { QoL } & \text { Quality of life }\end{array}$

\section{Introduction}

Ulcerative colitis (UC) is an inflammatory bowel disease (IBD) affecting the colonic mucosa spreading in a distal to proximal distribution. About twelve percent of UC patients will require colectomy at some point during their lifetime [1]. After the colectomy, patients can choose to keep the end ileostomy permanently or to undergo a restorative procedure. Depending on the circumstances, patients opting for a restorative procedure may choose between an ileo-rectal anastomosis (IRA) or proctectomy with construction of an 
ileal pouch-anal anastomosis (IPAA) [2]. Since the introduction in the late 1970s, procto-colectomy with IPAA has been the gold standard in most parts of the world $[3,4]$ despite reports of reduced fertility, impaired sexual function, and quality of life after IPAA [5-7]. It is still debated whether IRA is a safe alternative to IPAA due to the risk of recurrent proctitis and dysplasia or rectal cancer.

Recently, a Swedish population-based cohort study has concluded that despite a standardized incidence ratio for rectal cancer of 8.7 after IRA, the absolute risk of rectal cancer was only $1.6 \%$ after 10 years. This risk could possibly be further reduced through better selection of patients in combination with regular endoscopic surveillance and topical anti-inflammatory treatment. Moreover, some surgeons prefer IRA as a viable alternative to IPAA in UC patients [8] in order to postpone pelvic surgery to avoid possible side effects such as erectile dysfunction or reduced fecundability. In Sweden, IRA and IPAA are currently equally used as restorative procedures in UC patients [9].

A recent study concluded that IRA patients have better functional outcomes compared to IPAA patients if strict selection criteria were followed [10]. However, this conclusion was based on self-reported questionnaires without objective tests such as endoscopic evaluation or anal physiological studies.

In the present study, we used questionnaires to evaluate anorectal function and the quality of life (QoL) in UC patients with IRA and IPAA and assessed the impact of the macroscopic appearance at endoscopy and the microscopic grade of inflammation reported in biopsies. We hypothesize that IRA patients experience better function and QoL than patients restored with an IPAA.

\section{Methods}

Linköping University Hospital is a tertiary referral center for inflammatory bowel disease and colorectal surgery. Data about patients operated between 1992 and 2006 were extracted from the local IBD register. Complementary clinical data were collected from hospital records. We identified all patients with a diagnosis of UC who had gone through a restorative procedure with an IRA or an IPAA after a subtotal colectomy. Patients that had emigrated $(n=7)$ or died $(n=3)$ at the date of follow-up were excluded (Fig. 1). The remaining patients were invited by a letter to participate in the study. Those who accepted to participate were given further written and oral information by the responsible researchers. Then, informed consents were obtained from all individual participants included in the study. Participating patients were asked to complete three questionnaires on QoL and function and to undergo an endoscopic evaluation with mucosal biopsies. All biopsies were then evaluated regarding inflammatory activity and dysplasia by an experienced IBD pathologist.

The study was approved by the Linköping Regional Ethics Vetting Board (Dnr M127-07).

\section{Questionnaires}

Each participant completed the modified Öresland score, the validated Swedish form of the Short-Form Health Survey with 36 items (SF-36) and Short Health Scale (SHS) once when entering the study. Questionnaires and prepaid response envelopes were sent to the participants by mail. Participants were contacted only once. Some patients accepted to participate in the study and responded to the questionnaires but later withdrew their consent for the endoscopic part of the study.

The modified Öresland score is a validated questionnaire used for assessment of continence and bowel function and consists of 12 categories: the number of day and night time bowel motions, the presence of urgency, evacuation difficulties, day and night time soiling, perianal soreness, use of protective pads, dietary restrictions, frequency of medication use, and the presence of social impairment. Each item is scored as either $0 / 1$ or $0 / 1 / 2$, and the sum of all items ranges from 0 to 15 . A lower score indicates better function, and scores of eight or higher are considered associated with poor pouch function [11].

The Swedish version of $S F-36$ is a self-administered, wellvalidated indicator of overall health status divided into eight areas: vitality, bodily pain, physical function, general health perceptions, role limitations due to physical problems, role limitations due to emotional problems, social function, and mental health. Mental health is reported as the transition occurring in the last year. The score for each area is calculated from weighted sums. Scores range from 0 (worst possible health) to 100 (best possible health) [12].

SHS is a QoL questionnaire designed to measure the patients' subjective experience of how IBD influences four health dimensions: symptom burden, social function, disease-related anxiety, and sense of general well-being (Supplementary notes: Fig. 1). Responses are scored on a 100$\mathrm{mm}$ visual analogue scale (VAS) with individual scores for each of the four dimensions, zero representing the best score and 100 the worst. The SHS has been validated for both UC [13] and Crohn's disease [14].

\section{Endoscopic Evaluation}

All patients were invited to undergo an endoscopic evaluation of the rectal or ileal pouch mucosa, respectively. All endoscopies were performed within three weeks of answering the questionnaires (Fig. 1). The macroscopic grade of inflammation was assessed and reported using the 


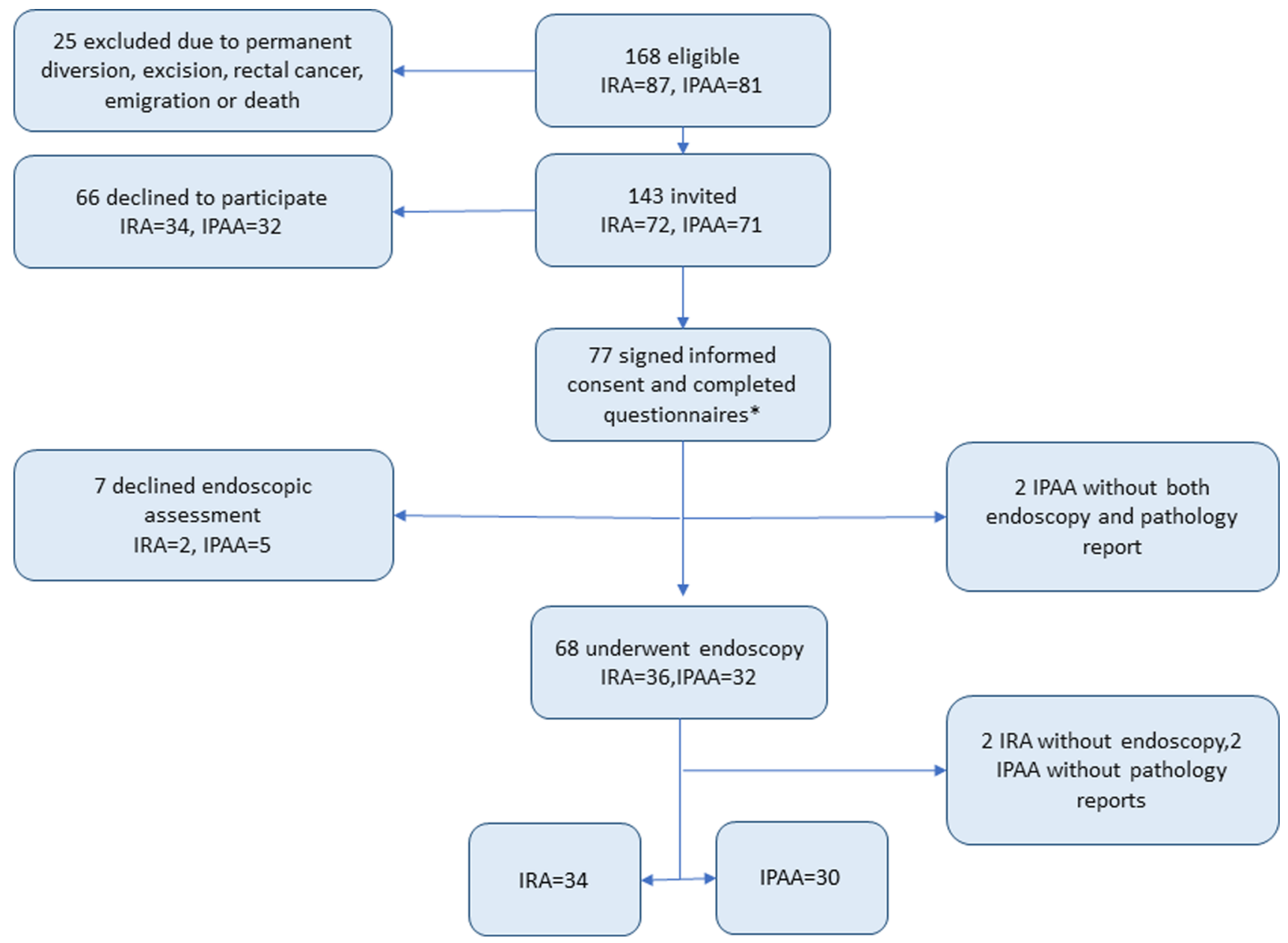

*Data of the 77 patients were included in demographic table and the questionnaire analyses. However, only 64 who had both an endoscopy report and a pathology report were included for the test of associations

Fig. 1 Flowchart of the included study participants

Baron-Ginsberg score (BG score) [15] for both IRA and IPAA patients. BG score is originally intended for evaluation of the rectal mucosa in UC.

Multiple biopsies were taken from the ileum, anastomotic site, upper rectum, and ampulla in case of IRA and from the ileum proximal of the pouch, from upper and lower parts of the pouch itself, and the rectal cuff in case of IPAA. The pathologist reported the grade of inflammation from all segments, coded as no, mild, moderate, or severe inflammation. A global grade of inflammation was estimated for each patient from the highest grade of inflammation found in any part of the reservoir or rectum. Dysplasia was reported as no, low-grade, or high-grade dysplasia. A few polyps were found and reported on the pathology report.

\section{Outcome}

The primary outcome was to compare function and QoL (SF-36, SHS and Öresland) between patients being restored with IRA or IPAA after colectomy.

The secondary outcome was to explore the association between the grades of inflammation, detected on both endoscopic and histopathologic, with bowel function and continence.

\section{Statistical Analysis}

Some patients did not answer all the questions of the questionnaires of the SF-36. If less than one-third of a questionnaire was left unanswered, the missing items scores were calculated through person-specific mean score calculated based on non-missing scores [16]. If more than one-third of the questions were left unanswered, the questionnaire 
Table 1 Demographics of the studied population

\begin{tabular}{lccc}
\hline & IRA $(n=38)$ & IPAA $(n=39)$ & $p$ value \\
\hline Males $[n(\%)]$ & $29(52.7)$ & $26(47.3)$ & 0.352 \\
Age at ulcerative colitis diagnosis & $25.8(6.4-57.2)$ & $25.2(9.1-42.0)$ & 0.910 \\
Age at reconstructive surgery & $33.1(10.3-75.2)$ & $35.2(18.9-58.9)$ & 0.424 \\
Endoscopy within study $[n(\%)]$ & $36(94.7 \%)^{+}$ & $32(82.1 \%)^{*}$ & 0.224 \\
Age at endoscopy within study & $45.9(22.3-86.2)$ & $51.3(29.4-74.7)$ & 0.145 \\
Interval between operation and study & $11.7(3.5-18.0)$ & $13.5(4.9-19.4)$ & 0.145 \\
$\quad$ endoscopy & & & \\
\hline
\end{tabular}

Time in years presented as median/range when not otherwise indicated. IPAA: Ileal pouch-anal anastomosis. IRA: Ileo-rectal anastomosis. Wilcoxon ranksum test was used

*Five IPAA declined endoscopic evaluation, two IPAA with missing endoscopy report, and two more with missing pathology reports

${ }^{+}$Two IRA patients declined endoscopic evaluation, two more had missing endoscopy reports, but one of them had a pathology report was excluded from the analysis. Seven patients declined to undergo the macroscopic assessment at the endoscopy. Moreover, four endoscopy reports were missing of which one pathology report was found. Two more pathology reports were missing.

Median and interquartile range (IQR) were used for calculating the general demographics and the total score of Öresland score, while mean and range were used in SF-36 and SHS.

Wilcoxon rank sum test was used to compare the test for demographic difference between groups as well as differences in the outcome of questionnaires between the patient groups (IRA and IPAA). Kendall-tau correlation coefficient analyzed the association of the grade of endoscopic inflammation, expressed by the Baron-Ginsberg score as well as the histopathologic grading of inflammation, with the function and QoL, expressed as the Öresland score, SF-36, and the SHS. Separate analyses were performed for IRA and IPAA patients. The strength of association was analyzed by ordered logistic regression and expressed as odds ratio (OR). Tests were considered statistically significant if the $p$ value was $<0.05$.

All analyses were performed using Stata 15 (StataCorp. 2015. Stata Statistical Software: Release 15. College Station, TX: StataCorp LP).

\section{Results}

The questionnaires were sent to 143 eligible patients. Seventy-seven patients (53.9\%) accepted to participate, 38 (49.4\%) with an IRA, and 39 (50.6\%) with an IPAA (Fig. 1). Among the patients with IPAA, two of the patients were later diagnosed with Crohn's disease and one with PSC. Sixty-eight patients (88.3\%) underwent endoscopic evaluation. The median follow-up after the restorative procedure was performed was 12.1 (range $3.5-19.4$, p value $=0.145)$ years (Table 1).

\section{Öresland Score}

Patients with IRA had better function according to the Öresland score with a median overall score of 3 (IQR 2-5) compared to 10 (IQR 5-15) for IPAA $(p<0.001)$ (Fig. 2). Only three $(7.9 \%)$ IRA patients had a total Öresland score $\geq 8$ compared to $21(53.8 \%)$ IPAA patients $(p<0.001)$.

The IRA patients had significantly fewer bowel movements with a median of 5 (range 1-13) compared to the IPAA patients with a median of 7 (range 3-14) $(p=0.002)$. More than six daytime bowel movements were reported by only five (13.2\%) IRA patients compared to $18(46.2 \%)$ IPAA patients $(p=0.002)$. Three IRA patients $(7.9 \%)$ had problems with nighttime soiling compared to $12(30.8 \%)$ IPAA patients $(p=0.012)$, and ten $(26.3 \%)$ IRA patients suffered from perianal soreness compared to 28 (71.8\%) IPAA patients $(p=0.001)$. Dietary restrictions were reported by nine IRA patients $(23.7 \%)$ compared with twenty IPAA patients $(51.3 \%)(p=0.013)$.

On the other hand, thirteen IRA patients (34.2\%) showed a trend toward more urgency compared to six (15.4\%) IPAA patients ( $p=0.057)$, and the number of IRA patients who received medications (occasionally or continuously) was $32(84.2 \%)$ compared to $22(56.4 \%)$ of the IPAA patients $(p=0.008)$. Twenty-eight IRA patients received mesalamine alone or combined with another medication (six patients with different preparations of topical steroids).

Stool frequency nighttime, daytime soiling, evacuation difficulties, social handicap, as well as the need to use protective pads were similar in IRA and IPAA patients. 
Fig. 2 Boxplots of the medians and CI 95\% of Öresland score in UC patients operated with IRA and IPAA

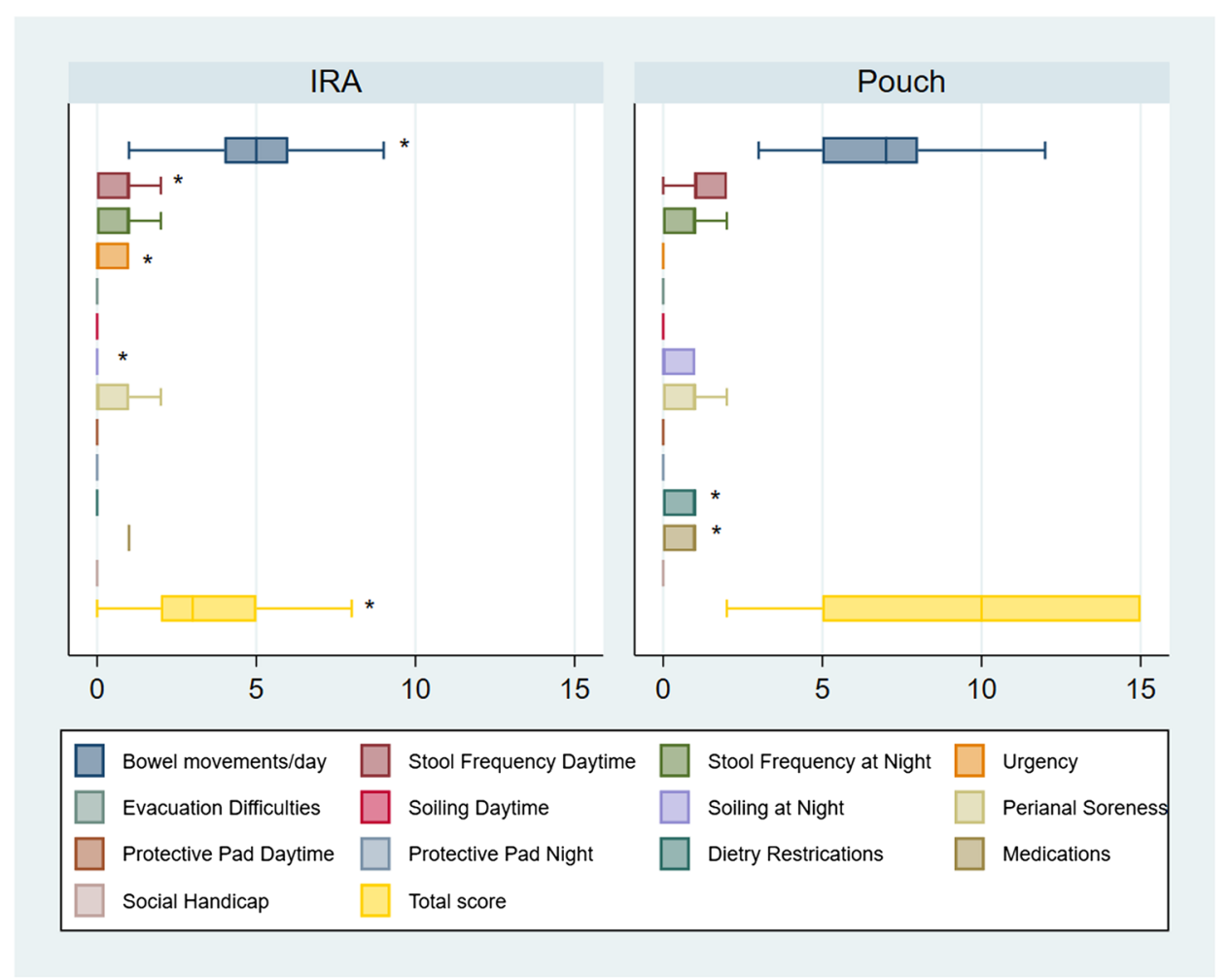

Zero score is the best possible score, and 15 is the worst possible score. ${ }^{*}$ p value $<0.05$. The IRA patients had significantly fewer bowel movements with a median of 5 (range 1-13) compared to the IPAA patients with a median of 7 (range $3-14)(p=0.002)$. More than six daytime bowel movements were reported by only five $(13.2 \%)$ IRA patients compared to $18(46.2 \%)$ IPAA patients $(\mathrm{p}=0.002)$. Three IRA patients $(7.9 \%)$ had problems with nighttime soiling compared to $12(30.8 \%)$ IPAA patients $(\mathrm{p}=0.012)$ and ten $(26.3 \%)$ IRA patients suffered from perianal soreness compared to $28(71.8 \%)$ IPAA patients $(\mathrm{p}=0.001)$. Dietary restrictions were reported by nine IRA patients $(23.7 \%)$ compared with twenty IPAA patients $(51.3 \%)(\mathrm{p}=0.013)$

\section{SF-36}

The total score of SF-36 did not differ significantly between IRA and IPAA patients, but there were differences for specific items (Fig. 3). The mean SF-36 score for the role limitation due to physical problems was worse for IRA patients (77.6) compared to IPAA patients $(92.3)(p=0.043)$. There was also a trend toward worse transition of mental health in IRA patient with a mean of 74.2 in compared to 85.3 in IPAA $(p=0.053)$.

Physical functioning, bodily pain, general health perceptions, role limitation due to emotional problems, vitality, and social function did not differ between IRA and IPAA patients.

\section{SHS}

All SHS items were similar among the IRA and IPAA patients (supplementary notes, Fig. 1).

\section{Endoscopy and Biopsy Results}

On endoscopy, six (18\%) IRA patients had a BG score of two or higher, compared to 11 (37\%) IPAA patients $(p=0.273)$. On histopathology, 21 (60\%) IRA patients had moderate to severe inflammation in the rectum, whereas 24 (77\%) of the IPAA patients displayed histological signs of pouch inflammation ( $p=0.454$ ) (Table 2).

Five patients in our study had evidence of cellular changes in their biopsies: One IPAA patient had low-grade dysplasia in the ileum, two more IPAA patients had hyperplasia at the rectal cuff/reservoir junction, and one IPAA patient had squamous metaplasia at the anastomotic mucosa. On the other hand, only one IRA patient had low-grade dysplasia at the anastomotic site. The frequency of cellular changes did not differ between the two groups (Table 2).

We found no statistical difference in the occurrence of polyps between IRA and IPAA patients $(p=0.146)$. Eleven patients had polyps in their reservoirs: eight (22.2\%) IRA (three hyperplastic and five inflammatory polyps) and three (9.4\%) IPAA patients (inflammatory polyps). 
Fig. 3 Boxplots of the medians and $95 \%$ confidence interval of SF-36 in ulcerative colitis patients operated with IRA or IPAA

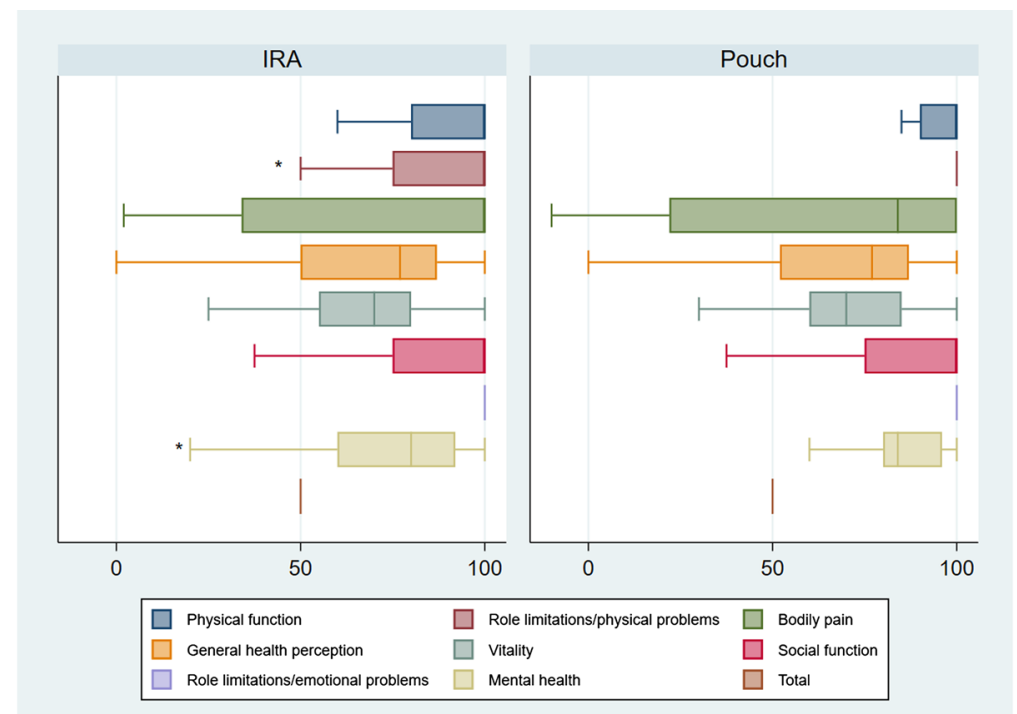

Zero represents the best possible and 100 the worst possible score. *Had a p value $<0.05$. The mean SF-36 score for the role limitation due to physical problems was worse for IRA patients (77.6) compared to IPAA patients (92.3) $(\mathrm{p}=0.043)$. There was also a trend toward worse transition of mental health in IRA patient with a mean of 74.2 in compared to 85.3 in IPAA $(\mathrm{p}=0.053)$
Table 2 Results of pathology reports: histological grades of inflammation, types and site of dysplasia, and number of polyps

\begin{tabular}{|c|c|c|c|}
\hline & IRA & IPAA & $p$ value \\
\hline Endoscopy* & $n=34$ & $n=30$ & 0.173 \\
\hline \multicolumn{4}{|l|}{ Baron-Ginsberg score } \\
\hline 0 & 7 & 6 & \\
\hline 1 & 21 & 13 & \\
\hline 2 & 5 & 11 & \\
\hline 3 & 1 & 0 & \\
\hline Pathology reports ${ }^{+}$ & $n=35$ & $n=30$ & 0.091 \\
\hline \multicolumn{4}{|l|}{ Inflammatory grades } \\
\hline None & 4 & 0 & \\
\hline Mild & 10 & 6 & \\
\hline Moderate & 11 & 17 & \\
\hline Severe & 10 & 7 & \\
\hline Cellular changes & & & 0.099 \\
\hline Low-grade dysplasia & 1 at anastomosis & 1 at ileum & \\
\hline Hyperplasia & & 2 at rectal cuff & \\
\hline Squamous metaplasia & & 1 rectal mucosa & \\
\hline
\end{tabular}

*Missing endoscopy reports from two IRA. Missing both endoscopy and pathology report from two IPAA patients. So (IRA $n=34$, IPAA $n=30$ )

${ }^{+}$Found an additional IRA pathology report without endoscopy report. Missing pathology report from two IPAA patients. So (IRA $n=35$, IPAA $n=30$ )

\section{Association Between Mucosal Inflammation and Function}

There was a positive correlation between Öresland score and endoscopic grades of inflammation (BG score) for IPAA patients only (tau. $0.28, p=0.006$ ) but not for IRA patients (supplementary notes, Table 1). For the IPAA patients, the strength of the association was further analyzed with ordered logistic regression. This shows that the Öresland score is strongly impaired when BG score increases, with OR 1.3 (CI -0.6-3.2, $p=0.188$ ) for grade 1 to OR 3.4 (CI 0.8-6.1, $p=0.012$ ) for grade 2 .

By contrast, there was no correlation between the results of pathology reports and the total Öresland score neither in IRA $(p=0.740)$ nor IPAA $(p=0.197)$ patients. Nor was there any correlation between total scores of SF-36 or SHS and BG score and the results of pathology reports regarding grades of inflammation, cellular changes, or the presence of polyps for both surgeries (data not shown).

\section{Association Between Macroscopic and Microscopic Grades of Inflammation}

There was a weak correlation between grades of inflammation reported macroscopically using BG endoscopic score and microscopically through pathology reports (tau. 0.19, $p=0.01$ ) in all patients. When studying correlation between BG score and pathology reports in IPAA and IRA, respectively, there was a correlation in the previous (tau. 0.26 , $p=0.021$ ) but not the IRA group (tau. $0.16, p=0.125$ ). 


\section{Discussion}

IRA patients have a better function compared with IPAA patients evaluated with the Öresland score, except for increased urgency and more frequent use of medications. Additionally, in the SF-36 form IRA patients reported more limitations regarding their physical functioning as well as worse mental health during the last year after IRA compared to IPAA patients. Interestingly, there was a positive correlation between the Öresland functional score and BG score in IPAA patients, suggesting a role for low-grade pouch inflammation in differences in anorectal function.

A recent study evaluated the QoL using the Cleveland clinic global quality of life score (CCGQoL) in 22 IRA patients age and sex matched to 66 IPAA patients $[17,18]$. The IRA patients reported fewer bowel movements, less night seepage, but they suffered from increased urgency, all in line with our results. Also, in accordance with the present study, IRA and IPAA patients reported similar postoperative QoL.

Another recent study used the validated Italian version of the CCGQoL to compare IRA and IPAA patients after restorative colectomy for UC, reporting higher QoL for IRA compared with IPAA patients [10]. Finally, a recent review of QoL of IBD patients concluded that IRA patients do not risk worse function than IPAA patients [19].

Several factors can cause variation in pouch function. Sunde et al. reported significantly different pouch volumes at first sensation, urge and discomfort with higher volumes, as well as a tendency toward shorter rectal cuff in the wellfunctioning pouch group. The patients with poorly functioning IPAA had a higher prevalence of inflammation, both macro- and microscopically [20]. Other studies related good pouch function to a higher compliance of the pouch wall as well as better sphincter functions measured with manometric studies [21, 22]. Since no manometric studies were performed in our study, we could not comment on neither volume differences inside the pouches nor on the sphincter functions. We could only investigate the possible association between the questionnaires and the presence of macro- and microscopic inflammation. All IPAA patients included in our study had a stapled anastomosis with a proved better function than hand-sewn pouches. Unfortunately, the length of the rectal cuff was not recorded in all patients so its impact could not be assessed [23].

In the present study, moderate to severe inflammation was more commonly detected macroscopically during endoscopies in IPAA (36.7\%) than in IRA (17.6\%). On the other hand, microscopic inflammation was commonly seen in macroscopically uninflamed mucosa, which explains the poor correlation between the macroscopic and microscopic grading of inflammation in the IRA patients. We conclude that it could be true that there is a subclinical inflammation of the rectal mucosa, but it could only be detected microscopically.

Previous reports have found similar or less frequent microscopic inflammation than in the present study, $26 \%$ moderate to severe proctitis, and $34-75 \%$ inflammatory activity in pouches [20,24]. A possible explanation is that the same pathologist did not assess all biopsies. However, the same highly experienced pathologist assessed 26 out of 32 IPAA reservoir biopsies and 31 of 36 rectal biopsies. Other pathologists who were well experienced assessed the remaining specimens.

For IPAA patients, there was a week association between the grades of inflammation reported on endoscopic evaluation (BG score) and the results of pathology reports evaluated by pathologists. This suggests that BG score can be a functional tool for diagnosis of endoscopic inflammation in IPAA patients. Similar association could not be found in the study by Zhu et al. Endoscopic evaluation of pouches of asymptomatic UC patients detected that $22.5 \%$ of the sample $(N=138)$ showed abnormal endoscopic findings (e.g., inflammation, polyps, or dysplasia). The histological examination of all the patients' biopsies revealed that $70.3 \%$ had chronic pouchitis and $17.4 \%$ had an acute pouchitis [25]. Regarding the IRA group, we could not find an association between the inflammation reported on endoscopic evaluation (BG score) and the results of pathology reports evaluated by pathologists for IRA patients.

There was a strong association between impaired function according to the Öresland score and the increased severity of inflammation reported on BG score in IPAA patients. This may suggest that pouch patients with moderate to severe subclinical pouchitis are more prone to have worse pouch function, and that this may be an undermedicated patient group. Other possible causes for poor pouch functions were previously investigated $[20,26]$, and smaller pouch volume was associated with poor function. A recent study suggests that the prophylactic use of Sulfasalazine in UC patients getting an IPAA could significantly reduce the risk of pouchitis and prolong the pouchitis-free interval [40].

From our study and previous reports [10,27, 28], we can conclude that IRA functional outcomes are not worse than in IPAA, even in the presence of mild inflammation. At our unit, IRA patients receive prophylactic anti-inflammatory medication with topical amino-salicylic acid (5-ASA) as standard of care. This is a treatment that works well for proctitis and has a possible cancer protective effect [24, 27-29]. Such treatment is well tolerated with few side effects [30] that could lower the risk of rectal cancer at least for the first 10-15 years after IRA. Moreover, it could lower the risk of proctectomy in case of severe proctitis or diarrhea that occurs in 8 to $53 \%$ of patients [31-33]. In such case, an IPAA can be safely performed with no evidence 
of increased risk of failure of the secondary reconstruction [34]. A Kock's continent ileostomy is another option with a high level of patient satisfaction [35].

On the other hand, patients who suffer from chronic or relapsing pouchitis will require treatment with systemic anti-inflammatory drugs which are less tolerable and with more side effects [36]. In 10-15\% of cases, treatment fails to control severe inflammation with risk of subsequent pouch failure and even excision [37]. Another study reported $20.5 \%$ refractory pouchitis leading to pouch failure and excision [38]. In these cases, patients are more likely to end up with either a diversion of the pouch, or even excision, and a permanent ileostomy [39].

IRA patients did not show an increased risk of dysplasia or polyps compared with IPAA patients during the median of 12.1 years of follow-up in our study. This is in concordance with a previous national cohort reporting a risk of rectal cancer in IRA of $1.8 \%$ at a mean follow-up of 8.6 years and with a cumulative risk of $1.6 \%$ and $5.6 \%$ after 10 and 20 years after receiving the IRA, respectively [41]. Routine endoscopic evaluations with multiple biopsies after IRA proved to be valuable for evaluation of rectal mucosa and early detection of dysplasia/cancer [42]. The procedure itself is well tolerated and takes a short time to perform with a low complication rate. On the other hand, pouch endoscopy has been shown less valuable due to the very low risk of dysplasia and is at the time reserved for complicated or high-risk cases [5, 43].

In addition, an IRA operation requires less pelvic dissection, which means lower risk of pelvic infection or adhesions and a future better fecundability and fertility, for both men and women, than in IPAA patients [24, 44-46].

However, IRA should not be offered to UC patients with a concomitant PSC diagnosis, because they have 6 times increased risk of developing cancer in their rectal mucosa [1]. Otherwise, we assume that IRA patients do not risk worse functional outcomes if properly selected.

However, this study has some limitations. Patients were consecutively recruited without matching for age or sex, but there were no significant differences between the two groups. Additionally, some patients suffered from pouchitis when they participated in the study, leading to worse QoL perception. One explanation to that may be the patients who suffer from more symptoms are more likely to accept being included in the study. Additionally, some IPAA patients declined to perform endoscopic evaluation because it was not a routine check or because they did not suffer from symptoms of pouchitis. This could in turn lead to selection bias. Moreover, there was a long-time interval between the time of restorative surgery, with either an IRA or an IPAA, and the time of participation in the study. This may affect the function of the reconstruction because it gets impaired after a longer follow-up [47]. We believe this to be less of a problem in our study because the time of follow-up was similar in both groups and there were only 3 weeks' interval between answering the questionnaires and going through endoscopic evaluation.

To our knowledge, this is the only study that compared the results of the self-reported QoL questionnaires to the results of endoscopic evaluations and pathology reports of the biopsies obtained from UC patients reconstructed with either an IRA or an IPAA. This could point out the actual magnitude of the patients' problems regarding their QoL.

\section{Conclusion}

Although UC patients reconstructed with IRA had an overall better function than patients with IPAA, differences of the impact of reconstruction on their QoL were unremarkable in the end. There was no difference in the prevalence of both macro- and microscopic inflammation between IRA and IPAA patients. Moreover, a previous report noted that although the relative risk of rectal cancer in IRA patients is high (SIR 8.7), the absolute risk is low [41]. In the case of a failed IRA, patients will still be able to have a secondary restorative procedure with an IPAA or a Kock's pouch. Thus, we assume that IRA can be a valid surgical option if both patient and surgeon follow strict selection criteria. This could be further confirmed through a prospective study evaluating the functional outcome for post-colectomy UC patients, planned for reconstruction with an IRA or IPAA.

Acknowledgments Open access funding provided by Linköping University.

Author's contributions RN, PM, and PA initiated the study concept and design. RN and MO performed the acquisition of data. MA, RA, and KL carried out the statistical analysis. RA, KL, MA, and PM performed the analysis and interpretation of data. PM and MA drafted the manuscript. RA, KL, MA, PM, MO, PA, and JS were involved in the critical revision of the manuscript for intellectual content.

Funding Medical Research Council of Southeast Sweden. Funding was provided by Forskningsrådet i Sydöstra Sverige (Grant Nos. FORSS569811, FORSS-756661).

\section{Compliance with Ethical Standards}

Conflict of interest All the authors declare that they have no conflict of interest.

Ethical approval All procedures performed in studies involving human participants were in accordance with the ethical standards of the institutional and/or national research committee and with the 1964 Helsinki Declaration and its later amendments or comparable ethical standards.

Open Access This article is distributed under the terms of the Creative Commons Attribution-NonCommercial 4.0 International License (http://creativecommons.org/licenses/by-nc/4.0/), which permits any 
noncommercial use, distribution, and reproduction in any medium, provided you give appropriate credit to the original author(s) and the source, provide a link to the Creative Commons license, and indicate if changes were made.

\section{References}

1. Abdalla M, Landerholm K, Andersson P, et al. Risk of rectal cancer after colectomy for patients with ulcerative colitis: a national cohort study. Clin Gastroenterol Hepatol. 2016;17:1055-1060.

2. Andersson P, Söderholm JD. Surgery in ulcerative colitis: indication and timing. Dig Dis. 2009;27:335-340.

3. Magro F, Gionchetti P, Eliakim R, et al. Third European evidencebased consensus on diagnosis and management of ulcerative colitis. Part 1: definitions, diagnosis, extra-intestinal manifestations, pregnancy, cancer surveillance, surgery, and ileo-anal pouch disorders. J Crohn's Colitis. 2017;11:649-670.

4. Hahnloser D, Pemberton JH, Wolff BG, et al. Results at up to 20 years after ileal pouch anal anastomosis for chronic ulcerative colitis. Br J Surg. 2007;94:333-340.

5. Berndtsson I, Öresland T. Quality of life before and after proctocolectomy and IPAA in patients with ulcerative proctocolitis-a prospective study. Colorectal Dis. 2003;5:173-179.

6. Rajaratnam SG, Eglinton TW, Hider P, et al. Impact of ileal pouch-anal anastomosis on female fertility: meta-analysis and systematic review. Int J Colorectal Dis. 2011;26:1365-1374.

7. Leowardi C, Hinz U, Tariverdian M, et al. Long-term outcome 10 years or more after restorative proctocolectomy and ileal pouch-anal anastomosis in patients with ulcerative colitis. Langenbecks Arch Surg. 2010;395:49-56.

8. Myrelid P, Øresland T. A reappraisal of the ileo-rectal anastomosis in ulcerative colitis. J Crohn's Colitis. 2015;9:433-438.

9. Nordenvall C, Myrelid P, Ekbom A, et al. Probability, rate and timing of reconstructive surgery following colectomy for inflammatory bowel disease in Sweden: a population-based cohort study. Colorectal Dis. 2015;17:882-890.

10. Tonelli F, Di Martino C, Giudici F. Could total colectomy with ileorectal anastomosis be an alternative to total proctocolectomy with ileal pouch-anal anastomosis in selected ulcerative colitis patients? Gastroenterol Res Pract. 2016;2016:5832743.

11. Öresland $T$, Fasth $S$, Nordgren $S$, et al. The clinical and functional outcome after restorative proctocolectomy. Int J Colorectal Dis. 1989;4:50-56.

12. Sullivan M, Karlsson J. The Swedish SF-36 health survey III. Evaluation of criterion-based validity: results from normative population. J Clin Epidemiol. 1998;51:1105-1113.

13. Hjortswang H, Järnerot G, Curman B, et al. The Short Health Scale: a valid measure of subjective health in ulcerative colitis. Scand J Gastroenterol. 2006;41:1196-1203.

14. Stjernman H, Grännö C, Järnerot G, et al. Short health scale: a valid, reliable, and responsive instrument for subjective health assessment in Crohn's disease. Inflamm Bowel Dis. 2008;14:47-52.

15. Baron JH, Connell AM, Lennard-Jones JE. Variation between observers in describing mucosal appearances in proctocolitis. $\mathrm{Br}$ Med J. 1964;1:89-92.

16. Sullivan M, Karlsson J, Ware JE. The Swedish SF-36 Health Survey-I. Evaluation of data quality, scaling assumptions, reliability and construct validity across general populations in Sweden. Soc Sci Med. 1995;41:1349-1358.

17. da Luz Moreira A, Kiran RP, Lavery I. Clinical outcomes of ileorectal anastomosis for ulcerative colitis. Br J Surg. 2010;97:65-69.
18. da Luz Moreira A, Lavery IC. Ileorectal anastomosis and proctocolectomy with end ileostomy for ulcerative colitis. Clin Colon Rectal Surg. 2010;23:269-273.

19. Bączyk G, Formanowicz D, Gmerek $€$, et al. Health-related quality of life assessment among patients with inflammatory bowel diseases after surgery—review. Przeglad Gastroenterologiczny. 2017;12:6-16.

20. Sunde ML, Ricanek P, Øresland T, et al. Determinants of optimal bowel function in ileal pouch-anal anastomosis-physiological differences contributing to pouch function. Scand J Gastroenterol. 2018;53:8-14.

21. Oresland T, Fasth $\mathrm{S}$, Nordgren $\mathrm{S}$, et al. Pouch size: the important functional determinant after restorative proctocolectomy. $\mathrm{Br} \mathrm{J}$ Surg. 1990;77:265-269.

22. Rink AD, Kneist W, Radinski I, et al. Differences in ano-neorectal physiology of ileoanal and coloanal reconstructions for restorative proctectomy. Colorectal Dis. 2010;12:342-350.

23. Lovegrove RE, Constantinides VA, Heriot AG, et al. A comparison of hand-sewn versus stapled ileal pouch anal anastomosis (IPAA) following proctocolectomy: a meta-analysis of 4183 patients. Ann Surg. 2006;244:18-26.

24. Andersson P, Norblad R, Söderholm JD, et al. Ileorectal anastomosis in comparison with ileal pouch anal anastomosis in reconstructive surgery for ulcerative colitis-a single institution experience. J Crohns Colitis. 2014;8:582-589.

25. Zhu H, Wu X, Queener E, et al. Clinical value of surveillance pouchoscopy in asymptomatic ileal pouch patients with underlying inflammatory bowel disease. Surg Endosc. 2013;27:4325-4332.

26. Ben-Bassat $\mathrm{O}$, Tyler $\mathrm{AD}, \mathrm{Xu} \mathrm{W}$, et al. Ileal pouch symptoms do not correlate with inflammation of the pouch. Clin Gastroenterol Hepatol. 2014;12:831-837.e2.

27. Borjesson L, Lundstam U, Öresland T, et al. The place for colectomy and ileorectal anastomosis: a valid surgical option for ulcerative colitis? Tech Coloproctol. 2006;10:237-241. (discussion 241).

28. Myrelid P, Oresland T. A reappraisal of the ileo-rectal anastomosis in ulcerative colitis. J Crohns Colitis. 2015;9:433-438.

29. Pinczowski D, Ekbom A, Baron J, et al. Risk factors for colorectal cancer in patients with ulcerative colitis: a case-control study. Gastroenterology. 1994;107:117-120.

30. Christophi GP, Rengarajan A, Ciorba MA. Rectal budesonide and mesalamine formulations in active ulcerative proctosigmoiditis: efficacy, tolerance, and treatment approach. Clin Exp Gastroenterol. 2016;9:125-130.

31. Juviler A, Hyman N. Ulcerative colitis: the fate of the retained rectum. Clin Colon Rectal Surg. 2004;17:29-34.

32. Leijonmarck CE, Löfberg R, Öst $\AA$, et al. Long-term results of ileorectal anastomosis in ulcerative colitis in Stockholm County. Dis Colon Rectum. 1990;33:195-200.

33. Melville DM, Ritchie JK, Nicholls RJ, et al. Surgery for ulcerative colitis in the era of the pouch: the St Mark's Hospital experience. Gut. 1994;35:1076-1080.

34. Landerholm K, Abdalla M, Myrelid P, et al. Survival of ileal pouch anal anastomosis constructed after colectomy or secondary to a previous ileorectal anastomosis in ulcerative colitis patients: a population-based cohort study. Scand J Gastroenterol. 2017;52:1-5.

35. Mathis KL, Dozois EJ, Larson DW, et al. Outcomes in patients with ulcerative colitis undergoing partial or complete reconstructive surgery for failing ileal pouch-anal anastomosis. Ann Surg. 2009;249:409-413.

36. Gionchetti P, Amadini C, Rizzello F, et al. Review article: treatment of mild to moderate ulcerative colitis and pouchitis. Aliment Pharmacol Ther. 2002;16:13-19.

37. Persborn M, Gerritsen J, Wallon C, et al. The effects of probiotics on barrier function and mucosal pouch microbiota during maintenance treatment for severe pouchitis in patients with ulcerative colitis. Alimentary Pharmacol Therapeut. 2013;38:772-783. 
38. Fazio VW, Ziv Y, Church JM, et al. Ileal pouch-anal anastomoses complications and function in 1005 patients. Ann Surg. 1995;222:120-127.

39. Shawki S, Belizon A, Person B, et al. What are the outcomes of reoperative restorative proctocolectomy and ileal pouch-anal anastomosis surgery? Dis Colon Rectum. 2009;52:884-890.

40. Scaioli E, Sartini A, Liverani E, et al. Sulfasalazine in prevention of pouchitis after proctocolectomy with ileal pouch-anal anastomosis for ulcerative colitis. Dig Dis Sci. 2017;62:1016-1024.

41. Abdalla M, Landerholm K, Andersson P, et al. Risk of rectal cancer after colectomy for patients with ulcerative colitis-a national cohort study. Clin Gastroenterol Hepatol. 2017;15:1055-1060.

42. Shuno Y, Hata K, Sunami E, et al. Is surveillance endoscopy necessary after colectomy in ulcerative colitis? ISRN Gastroenterol. 2011;2011:509251.

43. Mark-Christensen A, Erichsen R, Brandsborg S, et al. Long-term risk of cancer following ileal pouch-anal anastomosis for ulcerative colitis. J Crohns Colitis. 2018;12:57-62.
44. Waljee A, Waljee J, Morris AM, et al. Threefold increased risk of infertility: a meta-analysis of infertility after ileal pouch anal anastomosis in ulcerative colitis. Gut. 2006;55:1575-1580.

45. Mortier PE, Gambiez L, Karoui M, et al. Colectomy with ileorectal anastomosis preserves female fertility in ulcerative colitis. Gastroenterol Clin Biol. 2006;30:594-597.

46. Shin T, Okada H. Infertility in men with inflammatory bowel disease. World J Gastrointest Pharmacol Therapeutics. 2016;7:361-369.

47. Bengtsson J, Börjesson L, Lundstam U, et al. Long-term function and manovolumetric characteristics after ileal pouch-anal anastomosis for ulcerative colitis. Br J Surg. 2007;94:327-332.

Publisher's Note Springer Nature remains neutral with regard to jurisdictional claims in published maps and institutional affiliations.

\title{
Affiliations
}

\author{
Maie Abdalla ${ }^{1,2}$ (1) $\cdot$ Rickard Norblad ${ }^{3} \cdot$ Malin Olsson $^{3} \cdot$ Kalle Landerholm $^{4} \cdot$ Peter Andersson ${ }^{5,6} \cdot$ \\ Johan D. Söderholm ${ }^{3} \cdot$ Roland Andersson ${ }^{4} \cdot$ Pär Myrelid ${ }^{3}$ \\ Rickard Norblad \\ rickard.nordblad@regionostergotland.se \\ Malin Olsson \\ malin.olsson@ regionostergotland.se \\ Kalle Landerholm \\ kalle.landerholm@rjl.se \\ Peter Andersson \\ Peter.Andersson@ regionostergotland.se \\ Johan D. Söderholm \\ johan.d.soderholm@liu.se \\ Roland Andersson \\ rolandersson@gmail.com \\ Pär Myrelid \\ 2 Department of General Surgery, Faculty of Medicine, Suez \\ Canal University, Ismailia, Egypt \\ 3 Department of Surgery, Linköping University Hospital \\ and Department of Clinical and Experimental Medicine, \\ Linköping University, Linköping, Sweden \\ 4 Department of Surgery, Ryhov County Hospital, Jönköping, \\ and Department of Clinical and Experimental Medicine, \\ Linköping University, Linköping, Sweden \\ 5 Department of Surgery and Department of Clinical \\ and Experimental medicine, Linköping University, \\ Nörrköping, Sweden \\ 6 International Medical Program, Center for Teaching \\ and Research in Disaster Medicine and Traumatology, \\ University Hospital, Linköping, Sweden
}

par.myrelid@liu.se

1 Department of Clinical and Experimental Medicine, Linköping University, 58183 Linköping, Sweden 